\title{
A test of the bosonic spinon theory for the triangular antiferro- magnet spectrum
}

\author{
A. Mezio, C. N. Sposetti, L. O. Manuel and A. E. Trumper \\ Instituto de Física Rosario (CONICET) and Universidad Nacional de Rosario, Boulevard 27 de Febrero 210 bis, \\ (2000) Rosario, Argentina
}

PACS 75.10.Jm - Quantized spin models, including quantum spin frustration

\begin{abstract}
We compute the dynamical structure factor of the spin- $\frac{1}{2}$ triangular Heisenberg model using the mean field Schwinger boson theory. We find that a reconstructed dispersion, resulting from a non trivial redistribution of the spectral weight, agrees quite well with the spin excitation spectrum recently found with series expansions. In particular, we recover the strong renormalization with respect to linear spin wave theory along with the appearance of roton-like minima. Furthermore, near the roton-like minima the contribution of the two spinon continuum to the static structure factor is about $40 \%$ of the total weight. By computing the density-density dynamical structure factor, we identify an unphysical weak signal of the spin excitation spectrum with the relaxation of the local constraint of the Schwinger bosons at the mean field level. Based on the accurate description obtained for the static and dynamic ground state properties, we argue that the bosonic spinon theory should be considered seriously as a valid alternative to interpret the physics of the triangular Heisenberg model.
\end{abstract}

Introduction. - During a long time the magnetic ground state of the spin- $\frac{1}{2}$ triangular Heisenberg model ( THM) has attracted the attention of many researchers, due to the possible realization of the resonating valence bond (RVB) ground state proposed by P. W. Anderson in 1973 [1. The revival of the RVB theory for the cuprates 2] prompted the investigations of quantum disordered ground states within large $N$ theories where the Heisenberg interaction is naturally written in terms of singlet bond operators and fractional spin- $\frac{1}{2}$ excitations , with bosonic or fermionic character [3]. The fermionic version leads to exotic disordered ground states [4] while the bosonic one allows to describe disordered and ordered ground states 5 by relating the magnetization with the condensation of bosons [6]. For this case, using gauge field theoretical arguments, it has been conjectured that, when short range spiral correlations are present in the disordered phases, the bosonic spinons would be in a deconfined regime [5]. Therefore, a broad two spinon continuum is expected in the spin excitation spectrum.

From the numerical side, instead, thanks to the enormous effort of the community to develop unbiased techniques [7-10], it has been firmly established that the ground state of the spin- $\frac{1}{2}$ THM is a robust $120^{\circ}$ Néel order. These numerical results precluded the fermionic version of the RVB theory, giving support to both the linear spin wave theory (LSWT) and the bosonic version of the RVB theory, namely the Schwinger boson theory. In fact, both theories agree quite well with numerical results on finite size systems [1112, although for spiral phases the singlet structure of the mean field Schwinger bosons theory does not recover the spin wave dispersion relation in the large $s$ limit [13. Consequently, linear spin wave theory seemed to capture the quantum and semiclassical features expected for a $120^{\circ}$ Néel ground state of the THM. However, recent series expansions studies [10,14 challenged LSWT, showing that for $s=\frac{1}{2}$ the functional form of the dispersion relation differs considerably (points of fig. 2) from that of LSWT (solid line of fig. 2). In particular, it was observed a strong downward renormalization of the high energy part of the spectrum along with the appearance of roton-like minima at the midpoints of edges of the hexagonal Brillouin zone (BZ) (B and D points of the inset of fig. 11). The authors argued that the differences with LSWT could be attributed, probably, to the presence of fermionic spinon excitations. Nevertheless, further spin wave studies 15 showed that, to first order in $1 / s$, there appear non trivial corrections to the linear spin wave dispersion due to the non collinearity of the ground state, giving a fairly accurate description of the series expansion 
results. However, magnons are not well defined for an ample region of the BZ [16]. Another question, regarding the spectrum of the THM, is the nature of the multiparticle continuum above the one magnon states. For instance, it is believed that the broad multiparticle continuum measured in the $\mathrm{Cs}_{2} \mathrm{CuCl}_{4}$ compound is better described by an interacting spinon picture than a magnon one [17. In this sense, given accurate predictions of the Schwinger boson theory for the static ground state properties of the THM 11, it is important to investigate whether the anomalous features of the spectrum found with series expansions can be captured, or not, by this alternative theory that naturally incorporates fractional spin- $\frac{1}{2}$ excitations.

In the present article we investigate the validity of the bosonic spinon theory to interpret the spin excitation spectrum of the spin- $\frac{1}{2}$ THM. Our main finding is that the mean field Schwinger boson theory (intensity curves of fig. 21), based on the two singlet operator scheme [18, reproduces qualitatively and quantitatively quite well the recent series expansions results. By computing the dynamical structure factor, we remarkably find that the expected spin excitation spectrum is recovered by a reconstruction resulting from a non trivial redistribution of the spectral weight located at the spinonic branches shifted by $\pm \frac{\mathbf{Q}}{2}$, where $\mathbf{Q}=\left(\frac{4}{3} \pi, 0\right)$ is the magnetic wave vector. By computing the density-density dynamical structure factor, we were able to identify, at the mean field level, the remnant weaker signal of the spectrum with the relaxation of the local constraint of the number of bosons. We also discuss the validity of the alternative mean field decoupling based on one singlet operator scheme.

Mean field Schwinger bosons approximation. In the Schwinger boson representation [3] the spin operators are expressed as $\hat{\mathbf{S}}_{i}=\frac{1}{2} \mathbf{b}_{i}^{\dagger} \vec{\sigma} \mathbf{b}_{i}$, with the spinor $\mathbf{b}_{i}^{\dagger}=\left(\hat{b}_{i \uparrow}^{\dagger}, \hat{b}_{i \downarrow}^{\dagger}\right)$ composed by the bosonic operators $\hat{b}_{i \uparrow}^{\dagger}$ and $\hat{b}_{i \downarrow}^{\dagger}$, and $\vec{\sigma}=\left(\sigma^{x}, \sigma^{y}, \sigma^{z}\right)$ the Pauli matrices. To fulfil the spin algebra the constraint of $2 s$ bosons per site, $\sum_{\sigma} \hat{b}_{i \sigma}^{\dagger} \hat{b}_{i \sigma}=2 s$, must be imposed. Then, the spin-spin interaction of the Heisenberg Hamiltonian can be written as

$$
\hat{\mathbf{S}}_{i} \cdot \hat{\mathbf{S}}_{j}=: \hat{B}_{i j}^{\dagger} \hat{B}_{i j}:-\hat{A}_{i j}^{\dagger} \hat{A}_{i j},
$$

where :: means normal order and the singlet bond operators are defined as $\hat{A}_{i j}^{\dagger}=\frac{1}{2} \sum_{\sigma} \sigma \hat{b}_{i \sigma}^{\dagger} \hat{b}_{j \bar{\sigma}}^{\dagger}$ and $\hat{B}_{i j}^{\dagger}=$ $\frac{1}{2} \sum_{\sigma} \hat{b}_{i \sigma}^{\dagger} \hat{b}_{j \sigma}$. We will briefly describe the main steps of the mean field while the details of the calculation can be found in our previous works 11, 18. Introducing a Lagrange multiplier $\lambda$ to impose the local constraint on average and performing a mean field decoupling of eq. (1), such as $A_{i j}=\left\langle\hat{A}_{i j}\right\rangle=\left\langle\hat{A}_{i j}^{\dagger}\right\rangle$ and $B_{i j}=\left\langle\hat{B}_{i j}\right\rangle=\left\langle\hat{B}_{i j}^{\dagger}\right\rangle$, the diagonalized mean field Hamiltonian results

$$
\hat{H}_{M F}=E_{\mathbf{g s}}+\sum_{\mathbf{k}} \omega_{\mathbf{k}}\left[\hat{\alpha}_{\mathbf{k} \uparrow}^{\dagger} \hat{\alpha}_{\mathbf{k} \uparrow}+\hat{\alpha}_{-\mathbf{k} \downarrow}^{\dagger} \hat{\alpha}_{-\mathbf{k} \downarrow}\right]
$$

where

$$
E_{\mathrm{gs}}=\frac{1}{2} \sum_{\mathbf{k}} \omega_{\mathbf{k}}+\lambda N\left(s+\frac{1}{2}\right)
$$

is the ground state energy and

$$
\omega_{\mathbf{k} \uparrow}=\omega_{\mathbf{k} \downarrow}=\omega_{\mathbf{k}}=\left[\left(\gamma_{\mathbf{k}}^{B}+\lambda\right)^{2}-\left(\gamma_{\mathbf{k}}^{A}\right)^{2}\right]^{\frac{1}{2}},
$$

is the spinon dispersion relation with geometrical factors, $\gamma_{\mathbf{k}}^{B}=\frac{1}{2} J \sum_{\delta} B_{\delta} \cos \mathbf{k} . \delta$ and $\gamma_{\mathbf{k}}^{A}=\frac{1}{2} J \sum_{\delta} A_{\delta} \sin \mathbf{k} . \delta$, and with the sums going over all the vectors $\delta$ connecting the first neighbours of a triangular lattice. The mean field parameters has been chosen real and satisfy the relations $B_{\delta}=B_{-\delta}$ and $A_{\delta}=-A_{-\delta}$. The ground state wave function of $\hat{H}_{M F}$ can be written in a Jastrow form [6],

$$
|\mathrm{gs}\rangle=\exp \left[\sum_{i j} f_{i j} \hat{A}_{i j}^{\dagger}\right]|0\rangle_{b},
$$

where $|0\rangle_{b}$ represents the vacuum of Schwinger bosons and the odd pairing function is defined as $f_{i j}=$ $\left(\frac{1}{N}\right) \sum_{\mathbf{k}} f_{\mathbf{k}} e^{\imath \mathbf{k}\left(\mathbf{r}_{i}-\mathbf{r}_{j}\right)}$, with $f_{\mathbf{k}}=-v_{\mathbf{k}} / u_{\mathbf{k}}$, and Bogoliubov coefficients $u_{\mathbf{k}}=\left[\frac{1}{2}\left(1+\frac{\gamma_{\mathbf{k}}^{B}+\lambda}{\omega_{\mathbf{k}}}\right)\right]^{\frac{1}{2}}$ and $v_{\mathbf{k}}=\imath \operatorname{sgn}\left(\gamma_{\mathbf{k}}^{A}\right)\left[\frac{1}{2}(-1+\right.$ $\left.\left.\frac{\gamma_{\mathbf{k}}^{B}+\lambda}{\omega_{\mathbf{k}}}\right)\right]^{\frac{1}{2}}$. The singlet bond structure of eq. (2) guarantees the singlet behavior of $|g s\rangle$. Even if the Lieb-Mattis theorem cannot be applied to non bipartite lattices, the singlet character of the ground state for cluster sizes with an even number of sites $N$ has been confirmed numerically 7,8. It should be noted, however, that $|g s\rangle$ is not a true RVB state because the constraint is only satisfied on average. Furthermore, by solving the self consistent mean field equations at zero temperature,

$$
\begin{aligned}
A_{\delta} & =\frac{1}{2 N} \sum_{\mathbf{k}} \frac{\gamma_{\mathbf{k}}^{A}}{\omega_{\mathbf{k}}} \sin \mathbf{k} . \delta \\
B_{\delta} & =\frac{1}{2 N} \sum_{\mathbf{k}} \frac{\left(\gamma_{\mathbf{k}}^{B}+\lambda\right)}{\omega_{\mathbf{k}}} \cos \mathbf{k} \cdot \delta \\
s+\frac{1}{2} & =\frac{1}{2 N} \sum_{\mathbf{k}} \frac{\left(\gamma_{\mathbf{k}}^{B}+\lambda\right)}{\omega_{\mathbf{k}}},
\end{aligned}
$$

it is found that as the system size $N$ increases the singlet ground state $|g s\rangle$ develops $120^{\circ}$ Néel correlations signalled by the minimum gap of the spinon dispersion located at $\pm \frac{\mathbf{Q}}{2}$, where $\mathbf{Q}=\left(\frac{4}{3} \pi, 0\right)$ is the magnetic wave vector [19]. As the spinon gap behaves as $\omega_{ \pm \frac{\mathrm{Q}}{2}} \sim 1 / N$, for large system sizes the singular modes of eq. (3) can be treated apart, analogously to a Bose condensation phenomena 6. In particular, the local magnetization $m(\mathbf{Q})$ can be derived from the last line of eq. (3), yielding the relation [20.

$$
\frac{1}{2 N} \frac{\left(\gamma_{\frac{\mathbf{Q}}{2}}^{B}+\lambda\right)^{2}}{\omega_{\frac{\mathbf{Q}}{2}}^{2}}=S(\mathbf{Q})=\frac{N}{2} m^{2}(\mathbf{Q}),
$$

where $S(\mathbf{k})=\sum_{\mathbf{R}} e^{\imath \mathbf{k} \cdot \mathbf{R}}\left\langle\mathbf{g s}\left|\hat{S}_{0} \cdot \hat{S}_{\mathbf{R}}\right| \mathrm{gs}\right\rangle$ is the static structure factor. Formally, it can be shown that in the thermodynamic limit $|g s\rangle$ is degenerated with a manifold of Bose 
Table 1: Energy and magnetization of the $120^{\circ}$ Néel ground state of the spin- $\frac{1}{2}$ Heisenberg antiferromagnet on the triangular lattice as obtained with mean field Schwinger bosons within one 22] $(A)$ and two 21] $(A B)$ singlet scheme; Gaussian fluctuations 11 above the $A B$ mean field $(A B+$ Fluct), Quantum Monte Carlo [8] (QMC), linear spin wave theory (LSWT) and non linear spin wave theory $(\mathrm{LSWT}+1 / s) 23$

\begin{tabular}{lll}
\hline \hline & $E / J N$ & $m$ \\
\hline$A$ & -0.7119 & 0.328 \\
$A B$ & -0.5697 & 0.275 \\
$A B+$ Fluct & -0.5533 & \\
QMC & $-0.5458(1)$ & $0.205(1)$ \\
LSWT & -0.5388 & 0.2387 \\
LSWT $+1 / s$ & -0.5434 & 0.2497 \\
\hline \hline
\end{tabular}

condensate ground states, each one corresponding to all the possible orientations, in spin space, of the $120^{\circ}$ Néel order. In the Schwinger boson language the condensate of the up/down bosons at $\pm \frac{\mathbf{Q}}{2}$ and the normal fluids of bosons corresponds to the spiralling magnetization $m(\mathbf{Q})$ and the zero point quantum fluctuations, respectively [6]. For the triangular lattice the present mean field approximation 21] gives a local magnetization $m=0.275$.

An alternative procedure, is to use the operator identity $: \hat{B}_{i j}^{\dagger} \hat{B}_{i j}:+\hat{A}_{i j}^{\dagger} \hat{A}_{i j}=S^{2}$, and write the spin-spin interaction (11) in terms of the singlet operator $\hat{A}_{i j}[3,22,24]$ :

$$
\hat{\mathbf{S}}_{i} \cdot \hat{\mathbf{S}}_{j}=-2 \hat{A}_{i j d}^{\dagger} \hat{A}_{i j}+S^{2} .
$$

Even if eqs. (1) and (4) are equivalent, the latter leads to a different mean field decoupling with parameters $A_{\delta}$ and $\lambda$ 22|24. In table 1it is shown the values of the ground state energy and magnetization for the THM obtained with the two mean field Schwinger boson decouplings along with Gaussian fluctuations [11, linear spin wave theory, non linear spin wave theory $(\mathrm{LSWT}+1 / s)$ [23] ; and quantum Monte Carlo 8] (QMC) results [25]. Even though it has not yet been calculated, we expect that Gaussian fluctuations above the mean field will reduce the magnetization, as has already been found for the spin stiffness in the THM 11. From table 1 it is seen that the two singlet scheme describe quantitatively better the static properties of the THM.

\section{Dynamical structure factor. -}

Spin-spin correlation functions. We study the spectrum through the dynamical structure factor at $T=0$, defined as

$$
S^{\alpha \alpha}(\mathbf{k}, \omega)=\sum_{n}\left|\left\langle\operatorname{gs}\left|\hat{\mathbf{S}}_{\mathbf{k}}^{\alpha}(0)\right| n\right\rangle\right|^{2} \delta\left(\omega-\left(\epsilon_{n}-E_{\mathbf{g s}}\right)\right),
$$

where $\alpha$ denotes $x, y, z,|n\rangle$ are the excited states, and $\hat{\mathbf{S}}_{\mathbf{k}}^{\alpha}$ is the Fourier transform of $\hat{\mathbf{S}}_{i}^{\alpha}$. As we work on finite systems the $S U(2)$ symmetry is not broken explicitly and $S^{x x}=S^{y y}=S^{z z}$ (in what follows the $\alpha \alpha$ indices are discarded). A straightforward calculation leads to the expression

$$
S(\mathbf{k}, \omega)=\frac{1}{4 N} \sum_{\mathbf{q}}\left|u_{\mathbf{k}+\mathbf{q}} v_{\mathbf{q}}-u_{\mathbf{q}} v_{\mathbf{k}+\mathbf{q}}\right|^{2} \delta\left(\omega-\left(\omega_{-\mathbf{q}}+\omega_{\mathbf{k}+\mathbf{q}}\right)\right),
$$

which satisfies the correct sum rule $\int \sum_{\mathbf{k} \alpha} S^{\alpha \alpha}(\mathbf{k}, \omega) d \omega=$ $N s(s+1)$.

As at the mean field level the triplet excitations are made of two spin- $\frac{1}{2}$ free spinons a broad two spinon continuum is expected. Nevertheless, as the $120^{\circ}$ long range Néel order is developed there can be distinguished three distinct contributions in the spectrum. Following the interpretation of the spectra of [26], it is instructive to split eq. (5) as

$$
S(\mathbf{k}, \omega)=S_{\mathbf{k}, \omega}^{\text {sing }}+S_{\mathbf{k}, \omega}^{\text {cont }},
$$

by using the fact that $u_{ \pm \frac{\mathbf{Q}}{2}}=\left|v_{ \pm \frac{\mathbf{Q}}{2}}\right| \sim\left(\frac{N m}{2}\right)^{\frac{1}{2}}$ and $\omega_{ \pm \frac{\mathbf{Q}}{2}} \sim$ 0 . For $\mathbf{k}= \pm \mathbf{Q}$, the spectrum is dominated by zero energy processes that create two spinons in the condensate. This gives rise to the magnetic Bragg peaks which, to leading order, behave as $S_{ \pm \mathbf{Q}, \omega}^{\text {sing }} \sim N m^{2} \delta(\omega)$. For $\mathbf{k} \neq \pm \mathbf{Q}$, the spectrum is dominated by low energy processes that create one spinon in the condensate and another one in the normal fluid. This gives rise to a double peaked signal proportional to $m$, represented by

$$
\begin{aligned}
S_{\mathbf{k}, \omega}^{\operatorname{sing}} & =\frac{m}{4}\left|\imath u_{\mathbf{k}+\frac{\mathrm{Q}}{2}}-v_{\mathbf{k}+\frac{\mathrm{Q}}{2}}\right|^{2} \delta\left(\omega-\omega_{\mathbf{k}+\frac{\mathrm{Q}}{2}}\right)+ \\
& +\frac{m}{4}\left|\imath u_{\mathbf{k}-\frac{\mathrm{Q}}{2}}+v_{\mathbf{k}-\frac{\mathrm{Q}}{2}}\right|^{2} \delta\left(\omega-\omega_{\mathbf{k}-\frac{\mathrm{Q}}{2}}\right) .
\end{aligned}
$$

Then, the shifted spinon dispersion $\omega_{\mathbf{k} \pm \frac{\mathrm{Q}}{2}}$ can be identified with the low energy physical magnetic excitations. Finally, at high energy, the spectrum is dominated by the processes of creating two spinons in the normal fluid. This gives rise to a broad continuum represented by

$$
S_{\mathbf{k}, \omega}^{c o n t}=\frac{1}{4 N} \sum_{\mathbf{q}}^{\prime}\left|u_{\mathbf{k}+\mathbf{q}} v_{\mathbf{q}}-u_{\mathbf{q}} v_{\mathbf{k}+\mathbf{q}}\right|^{2} \delta\left(\omega-\left(\omega_{-\mathbf{q}}+\omega_{\mathbf{k}+\mathbf{q}}\right)\right),
$$

where the prime means that sum goes over the triangular BZ except for $\mathbf{q}= \pm \frac{\mathbf{Q}}{2}$ or $\pm \frac{\mathbf{Q}}{2}-\mathbf{k}$.

In fig. 1 we have plotted eq. (5) for the $M$ point of the BZ (see inset of fig. 1). As noticed above, the low energy double peaked structure comes from $S_{\mathbf{k}, \omega}^{s i n g}$ while the high energy tail corresponds to the continuum $S_{\mathbf{k}, \omega}^{c o n t}$. In order to get the spectrum in the energy-momentum space we have plotted in fig. 2 the intensity curves of $S(\mathbf{k}, \omega)$ (eq. (5)) along the path shown in the inset of fig. 1. The yellow and red curves are the shifted spinon dispersion $\omega_{\mathbf{k} \mp \frac{\mathbf{Q}}{2}}$ of $S_{\mathbf{k}, \omega}^{\text {sing }}$ while the blue zone corresponds to $S_{\mathbf{k}, \omega}^{\text {cont }}$. In the figure we compare with the dispersion relations obtained with LSWT (solid line) and the recent series expansion calculations 14 (points). At low energies the dispersion agrees quite well with LSWT and series expansions, being the spectral weight mostly located around $\mathbf{k} \sim \pm \mathbf{Q}$ 


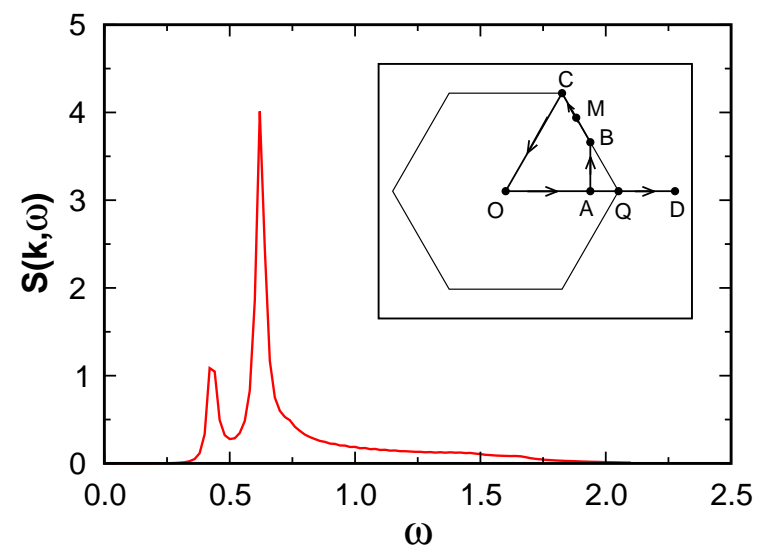

Fig. 1: Dynamical structure factor, $S(\mathbf{k}, \omega)$, for momentum $M=\left(\frac{5}{6} \pi, \frac{\sqrt{3}}{2} \pi\right)$. Inset: path of the triangular BZ along which the spectrum has been investigated. $O=(0,0), A=(\pi, 0), Q=$ $\left(\frac{4}{3} \pi, 0\right), D=(2 \pi, 0), B=\left(\pi, \frac{1}{\sqrt{3}} \pi\right)$, and $C=\left(\frac{2}{3} \pi, \frac{2}{\sqrt{3}} \pi\right)$. $\omega$ is measured in units of $J$.

(points $\mathrm{Q}$ and $\mathrm{C}$ ). In this regime the physical excitations correspond to long range transverse distorsions of the local magnetization which are correctly described by both, LSWT and mean field Schwinger bosons. At higher energies LSWT is not valid any more since the true spin excitations show a strong downward renormalization along with the appearance of roton-like minima (points). Remarkably, the mean field Schwinger boson theory predicts a non trivial redistribution of the spectral weight between the two spinon branches modulated by the form factor of eq. (5). The reconstructed dispersion, resulting from those pieces of spinon dispersion with the dominant spectral weight, reproduces quite well the series expansions results. In particular, the crossing of the spinon dispersions at points $B$ and $D$ can be identified with the roton-like minima observed in series expansions. Regarding the interpretation of the roton minima, the singlet bond structure of the Schwinger boson theory takes naturally into account the collinear spin fluctuations even in the presence of the $120^{\circ}$ Néel order of the THM. For instance, the roton minimum located at $B$ can be interpreted as the development of magnetic correlations modulated by the magnetic wave vector $\left(\pi, \frac{1}{\sqrt{3}} \pi\right)$ which corresponds to certain collinear correlations pattern, while the other two non equivalent midpoints of the edges of the hexagonal BZ corresponds to different collinear fluctuations patterns. In fact, if these fluctuations are favoured by introducing spatially anisotropic or second neighbours exchange interactions the roton minima soften, giving rise to the new Goldstone mode structure of the stabilized collinear ground state [21,27.

Performing the frequency integration it is possible to analyze the relative weight of the two spinon continuum (blue zone of fig. 2) to the static structure factor $S(\mathbf{k})$ 28. In fig. 3 we plot $S(\mathbf{k})$ with diverging peaks located at the expected magnetic wave vectors $\pm \mathbf{Q}$ (upper panel), along with the relative weight of the two spinon contin-

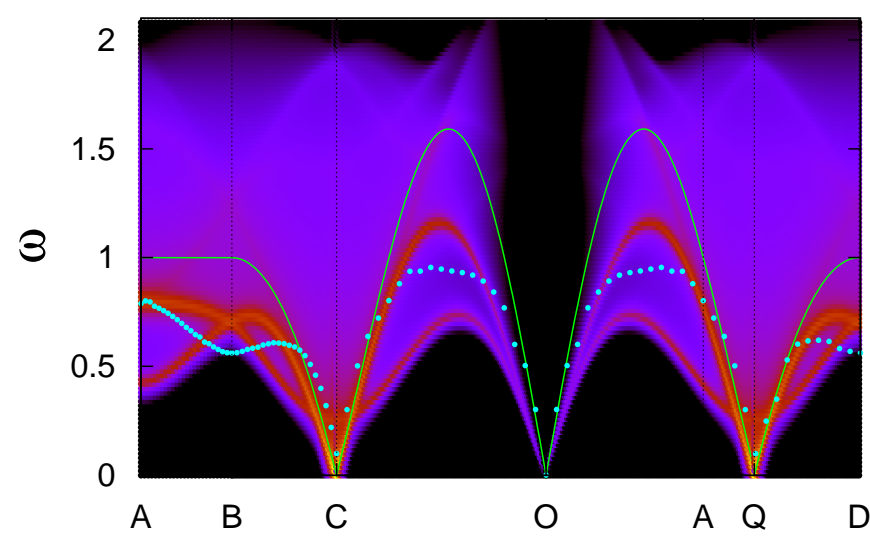

Fig. 2: Intensity curves for the dynamical structure factor, $S(\mathbf{k}, \omega)$, calculated with the mean field Schwinger bosons theory within the two singlet scheme. Solid green line and blue points are the dispersion relations obtained with LSWT and series expansions [14, respectively. The path along the BZ is shown in the inset of fig. 1

uum, $\int S_{\mathbf{k}, \omega}^{\text {cont }} / S(\mathbf{k}) d \omega$ (bottom panel). Interestingly, the contribution of the two spinon continuum to $S(\mathbf{k})$ is neglegible around $\pm \mathbf{Q}$ while outside their neighbourhood, and in particular at the roton position, the contribution to $S(\mathbf{k})$ is about $40 \%$.

Density-density correlation functions. The small peak of fig. 1 leads to the remnant weak signal of fig. 2 which can be traced back to the local density fluctuation of Schwinger bosons. In fact, to describe the physical Hilbert space of the spin operators the local constraint of the Schwinger bosons must be satisfied exactly, $\hat{\mathbf{S}}_{i}^{2}=\frac{n_{i}}{2}\left(\frac{n_{i}}{2}+1\right)$. Then, no fluctuations on the number of boson per site should be observed. However, since the constraint is taken into account on average there are unphysical spin fluctuations in $S(\mathbf{k}, \omega)$ coming from such density fluctuations. In order to identify them we have computed the density-density dynamical structure factor defined as

$$
N(\mathbf{k}, \omega)=\sum_{n}\left|\left\langle\operatorname{gs}\left|\hat{n}_{\mathbf{k}}(0)\right| n\right\rangle\right|^{2} \delta\left(\omega-\left(\epsilon_{n}-E_{\mathrm{gs}}\right)\right),
$$

where $\hat{n}_{\mathbf{k}}$ is the Fourier transform of the number of bosons per site, $\hat{n}_{i}=\sum_{\sigma} \hat{b}_{i \sigma}^{\dagger} \hat{b}_{i \sigma}$. A little of algebra leads to the expression

$N(\mathbf{k}, \omega)=\frac{1}{N} \sum_{\mathbf{q}}\left|u_{\mathbf{k}+\mathbf{q}} v_{\mathbf{q}}+u_{\mathbf{q}} v_{\mathbf{k}+\mathbf{q}}\right|^{2} \delta\left(\omega-\left(\omega_{-\mathbf{q}}+\omega_{\mathbf{k}+\mathbf{q}}\right)\right)$,

which is similar to eq. (5), except to the plus sign within the form factor. If we split the two spinon contributions as $N(\mathbf{k}, \omega)=N_{\mathbf{k}, \omega}^{\text {sing }}+N_{\mathbf{k}, \omega}^{\text {cont }}$ it is easy to show that the main signal is located again at the shifted spinon dispersions $\omega_{\mathbf{k} \mp \frac{\mathbf{Q}}{2}}$. But now, due to the different form factor, there is an important spectral weight transfer between such spinon dispersions. This is shown in fig. 4 where we have plotted the intensity curves of $N(\mathbf{k}, \omega)$ (eq. (6) ). It can be clearly observed that now the dominant signal is gapped 


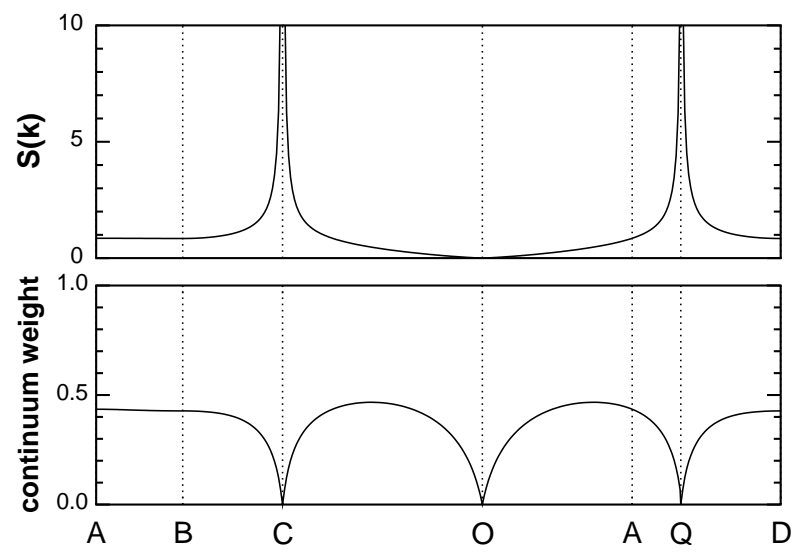

Fig. 3: Static structure factor (upper panel) and relative weight of the two spinon continuum, $\int S_{\mathbf{k}, \omega}^{\text {cont }} / S(\mathbf{k}) d \omega$, (bottom panel) along the same path of the BZ.

at $\mathrm{Q}$ and $\mathrm{C}$ points, while most of the spectral weight is located around $\mathbf{k} \sim 0$. Such a soft mode can be identified with a spurious tendency of the bosonic system to phase separation. Given the notable resemblance with the strong signal of $N(\mathbf{k}, \omega)$, we suggest that the low energy weak signal of figs. 1 and 2 could be ascribed with the unphysical density fluctuation effects which we expect to disappear once they are projected out. For the unfrustrated square lattice $\omega_{\mathbf{k}+\left(\frac{\pi}{2}, \frac{\pi}{2}\right)}=\omega_{\mathbf{k}-\left(\frac{\pi}{2}, \frac{\pi}{2}\right)}$ so both, the unphysical and the physical spin excitations, overlap in energy-momentum space, giving rise only to one low energy band in $S(\mathbf{k}, \omega)$ [3, 28].

Comparison with the one singlet scheme. So far we have found that the mean field Schwinger boson within the two singlet scheme reproduces quite well the series expansions spectrum. It is also interesting to compare with the predictions of the one singlet scheme, since it is widely used in the literature. The first difference is the incorrect sum rule $\int \sum_{\mathbf{k} \alpha} S^{\alpha \alpha}(\mathbf{k}, \omega) d \omega=\frac{3}{2} N s(s+1)$ which implies the well known $\frac{2}{3}$ factor of Arovas and Auerbach [3]. Furthermore, in fig. 5 we have computed $S(\mathbf{k}, \omega)$ after solving the corresponding self consistent equations for the parameters $A_{\delta}$, and $\lambda$. At very low energies the spectrum seems to be correct around points $C, O$ and $Q$. However, at higher energies it is impossible to discern a reconstructed dispersion that fit the series expansion results along the whole path of the BZ, besides the factor about 3 in the energy scale. Therefore, we conclude that the two singlet scheme turns out the proper framework to describe correctly the spectrum of the THM. Besides its quantitative accuracy, there are symmetry arguments that give further support to the two singlet scheme. In the literature, the one singlet scheme has been justified as the saddle point of a symplectic $S p(N)$ theory, originally adapted to extend previous large $N$ works 3 to non bipartite lattices 5 . More recently, however, Flint and Coleman 29] demonstrated that if the $\hat{B}_{i j}$ and $\hat{A}_{i j}$ operators are kept the corresponding large $N$ extension preserves the time reversal

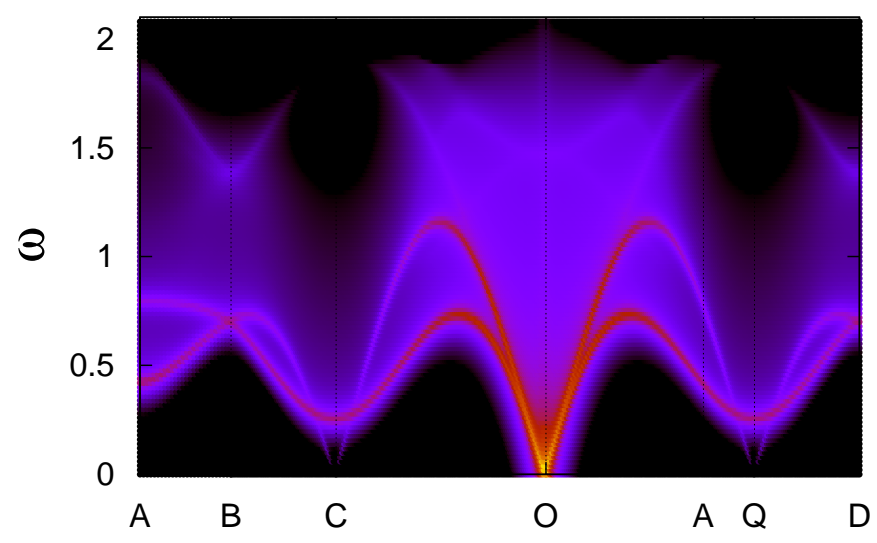

Fig. 4: Intensity curves for the density-density dynamical structure factor, $N(\mathbf{k}, \omega)$, calculated within the mean field Schwinger bosons based on the two singlet scheme. The path along the BZ is shown in the inset of fig. 1.

properties of the spins, in contrast to the $S p(N)$ theory. Finally, it is worth to stress that the two singlet scheme is the basis of the $Z_{2}$ spin liquid theory, specially formulated to describe magnetically disordered phases 30.

Conclusions. - We have demonstrated that the singlet structure of the mean field ground state along with the fractional character of the spin excitations of the Schwinger boson theory take naturally into account the anomalous excitations of the spin- $\frac{1}{2}$ triangular Heisenberg model recently observed [10,14. The appearance of the roton-like minima can be attributed to the tendency of the magnetic ground state to be correlated collinearly, even in the presence of $120^{\circ}$ Néel order. By computing the density-density dynamical structure factor, and thanks to the series expansion results, we were able for the first time to discern, at the mean field level, between the physical and the spurious fluctuations coming from the relaxation of the local constraint. A further investigation within the context of the Schwinger boson theory reveals that the correct description of the spectrum depends crucially on the mean field decoupling. In particular, the two singlet scheme turns out more appropriate than the one singlet scheme. Based on the accurate description of the ground state static properties 11. (see table 1) and in the light of the present results for the spectrum, we think that the bosonic spinon hypothesis should be considered seriously as an alternative viewpoint to interpret the physics of the triangular Heisenberg model. At the mean field level the triplet excitations consist of two spin- $\frac{1}{2}$ free spinons and, besides the low energy bands due to the onset of the long range order, there is a broad two spinon continuum, which could be related with the magnon decay found in the literature 23. In this sense, it would be important to improve the present mean field theory by deriving an effective interaction between spinons resulting from $1 / N$ corrections or a better implementation of the constraint. We would expect a picture of tightly bound spinons near the Goldstone modes while at high energies they would be weakly bound. 


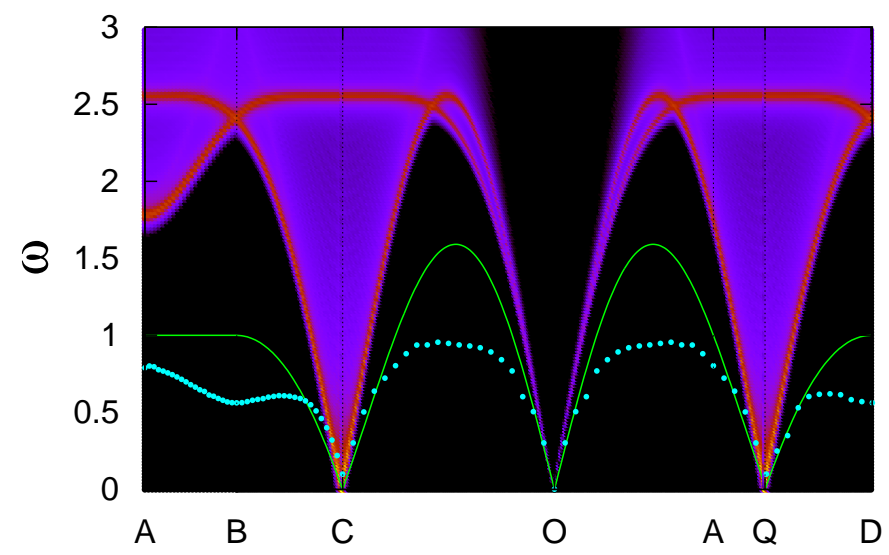

Fig. 5: Intensity curves for the dynamical structure factor calculated within the mean field Schwinger bosons based on the one singlet scheme. The path along the BZ is shown in the inset of fig. 1. Solid line and points are the same as in fig. 2

Work in this direction is in progress. Finally, we hope our present analysis in terms of bosonic spinons could help for a better understanding of the unconventional neutron scattering spectra of the $\mathrm{Cs}_{2} \mathrm{CuCl}_{4}$ compound [17].

$$
* * *
$$

We thank W. Zheng and R. Coldea for sending us their series expansions results, and C. Lhuillier and C. Batista for very useful discussions. This work was supported by PIP2009 under grant No. 1948.

\section{REFERENCES}

[1] Anderson P. W., Mater. Res. Bull., 8 (1973) 153; Fazekas P. And Anderson P. W., Philos. Mag., 30 (1974) 423.

[2] Anderson P. W., Science, 235 (1987) 1196.

[3] Auerbach A. And Arovas D. P., Phys. Rev. Lett., 61 (1988) 617; Arovas D. P. And Auerbach A., Phys. Rev. $B, 38$ (1988) 316.

[4] Affleck I. and Marston J. B., Phys. Rev. B, 37 (1988) 3774.

[5] Read N. And Sachdev S., Phys. Rev. Lett., 66 (1991) 1773; Sachdev S. And Read N., Int. J. Mod. Phys. B, 5 (1991) 219.

[6] Chandra P., Coleman P. And Larkin A. I., J. Phys. Condens. Matter, 2 (1990) 7933.

[7] Bernu B., Lhuillier C. And Pierre L., Phys. Rev. Lett., 69 (1992) 2590.

[8] Capriotti L., Trumper A. E. and Sorella S., Phys. Rev. Lett., 82 (1999) 3899.

[9] Huse D. A. And Elser V., Phys. Rev. Lett., 60 (1988) 2531; Leung P. W. And Runge K. J., Phys. Rev. B, 47 (1993) 5861; Kruger S. E., Darradi R., Richter J. and Farnell D. J. J., Phys. Rev. B, 73 (2006) 094404; White S. R. And Chernyshev A. L., Phys. Rev. Lett., 99 (2007) 127004.
[10] Zheng W., Fuaerestad J. O., Singh R. R. P., McKenZIE R. H. AND Coldea R., Phys. Rev. B, 74 (2006) 224420 .

[11] Manuel L. O., Trumper A. E. and Ceccatto H. A., Phys. Rev. B, 57 (1998) 8348.

[12] Lecheminant P., Bernu B., Lhuillier C. and Pierre L., Phys. Rev. B, 52 (1995) 9162; Trumper A. E., CApriotti L. And Sorella S., Phys. Rev. B, 61 (2000) 11529.

[13] Chandra P., Coleman P. and Ritchey L., Int. J. Mod. Phys. B, 5 (1991) 171.

[14] Zheng W., Fjaerestad J. O., Singh R. R. P., McKenZIE R. H. And Coldea R., Phys. Rev. Lett., 96 (2006) 057201.

[15] Starykh O. A., Chubukov A. V. and Abanov A. G., Phys. Rev. B, 74 (2006) 180403.

[16] Chernyshev A. L. And Zhitomirsky M. E., Phys. Rev. Lett., 97 (2006) 207202 .

[17] Coldea R., Tennant D. A. and Tylczynski Z., Phys. Rev. B, 68 (2003) 134424.

[18] Ceccatto H. A., Gazza C. J. and Trumper A. E., Phys. Rev. B, 47 (1993) 12329.

[19] Note that, in contrast to ref. [18, the self consistent equations (3) do not depend on $\mathbf{Q}$ explicitly because we have transformed as $\hat{b}_{\mathbf{k} \sigma}=\frac{1}{\sqrt{N}} \sum_{i} \hat{b}_{i \sigma} e^{\imath \mathbf{k} \cdot \mathbf{R}_{i}}$. Both procedures lead to the same results.

[20] Hirsch J. E. And Tang S., Phys. Rev. B, 39 (1989) 2850.

[21] Gazza C. J. and Ceccatto H. A., J. Phys.: Condens. Matter, 5 (1993) L135.

[22] Yoshioka D. and Miyazaki J., J. Phys. Soc. Jpn., 60 (1991) 614.

[23] Chernyshev A. L. And Zhitomirsky M. E., Phys. Rev. $B, 79,(2009) 144416$.

[24] Sachdev S., Phys. Rev. B, 45, (1992) 12377.

[25] A complete summary of the results for the THM derived by different methods can be found in ref. [10.

[26] Lefmann K. and Hedegård P., Phys. Rev. B, 50 (1994) 1074; Messio L., Cepas O. and Lhuillier C., Phys. Rev. $B, 81$ (2010) 064428 .

[27] Manuel L. O. and Ceccatto H. A., Phy. Rev. B, 60 (1999) 489; Trumper A. E., Phys. Rev. B, 60 (1999) 2987.

[28] Capriotti L., Läuchli A. And Paramekanti A., Phys. Rev. B, 72 (2005) 214433.

[29] Flint R. and Coleman P., Phys. Rev. B, 79 (2009) 014424.

[30] Wang F. and Vishwanath A., Phys. Rev. B, 74 (2006) 174423. 